\title{
CAN WE AFFORD TO WAIT? DESIGNING THE COLLIDER OF THE FUTURE
}

- Michael Benedikt and Frank Zimmermann - DOI: https://doi.org/10.1051/epn/2017401

- European Organisation for Nuclear Research (CERN) - CH-1211 Geneva, Switzerland - correspondence email: Michael.Benedikt@cern.ch

Designing a future circular collider is a next step in humanity's quest to explain the world. This effort is not only about striving for a profound understanding of nature, but also about creating an exciting perspective for future generations. 
- FIG. 2:

(a) Sketch of a future

80-100-km-long tunnel in the Geneva

area, which would allow for a $100-\mathrm{TeV}$,

energy-frontier proton collider and also, as a potentia intermediate step, a high-luminosity $\mathrm{e}^{+} \mathrm{e}$ collider serving as a

$\mathrm{W}, \mathrm{Z}, \mathrm{H}$ and $\mathrm{t} \overline{\mathrm{t}}$ factory. (Image credit: CERN)

(b) Schematic of

the underground infrastructure preliminary

layout. FCC study

envisages a $100 \mathrm{~km}$ in circumference tunnel, nearly four times the size of the present LHC (Image credit: CERN/FC(study)

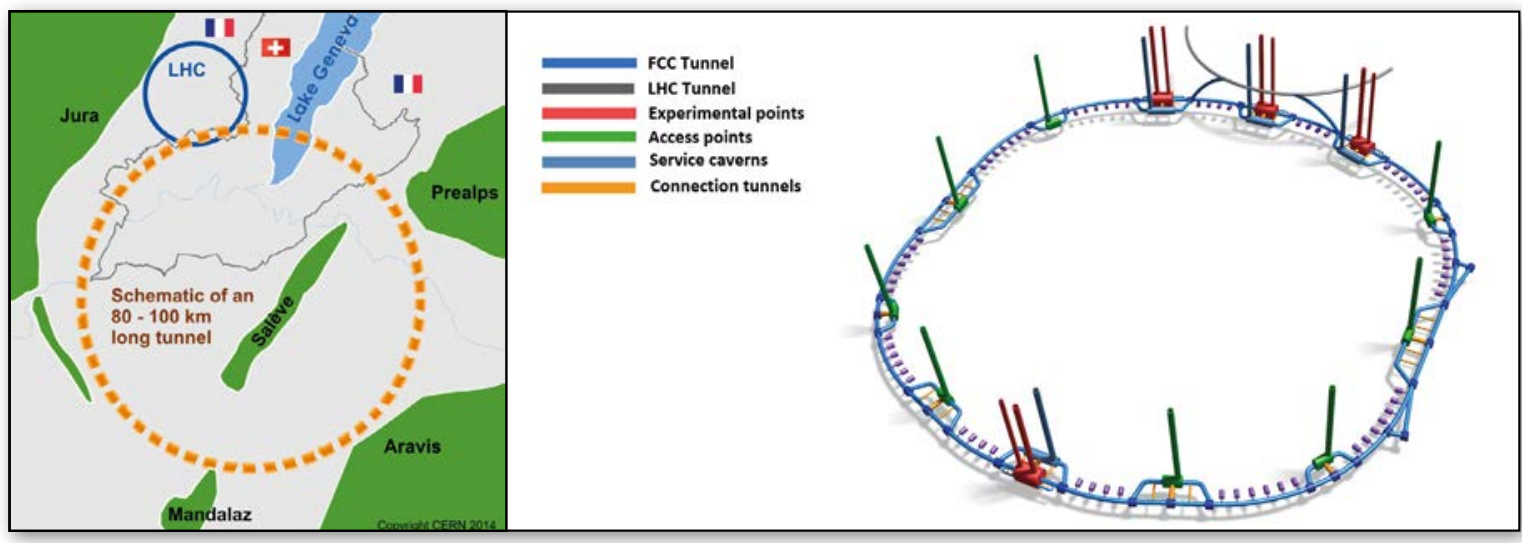

\section{A large-scale research} infrastructure for Europe

Presently we are testing our theories in the new energy regime opened up by the LHC. If new physics is uncovered at the LHC we will surely need to study it and further push the intensity and energy frontiers. But what if there is no sign of new physics at the LHC? In that case new experiments at higher energies but also of greater precision will be required to unravel the many unsolved mysteries of the Universe.

To prepare for either case, CERN has initiated the FCC study for a future large-scale research infrastructure centred on a new-generation circular hadron collider with a circumference of about 100 kilometres, able to reach proton-proton collision energies of $100 \mathrm{TeV}$ [5] - about 8 times above the LHC - and a corresponding energy in heavy-ion collisions. A future high-luminosity electron-positron collider, which could be housed in the same tunnel, is being considered as a possible first step [6]. A high-energy LHC upgrade is yet another option.

The FCC study explores the feasibility and reliability of all these possible future machines and associated options such as ion operation and lepton-hadron collisions (FCC-he); it also develops key-enabling technologies and examines the various physics opportunities. These efforts will culminate in a Conceptual Design Report and a cost review that will inform the next Update of the European Strategy for Particle Physics expected around 2020.

Investment in frontier research will reinforce and revive European competitiveness, employment, and prosperity, while preparing the next generation of accelerators. However, the FCC study extends well beyond Europe: it is organized as an international collaboration, bringing together as of today 116 institutes from 33 countries. This is in line with CERN's long standing tradition. LHC is but the latest example in a series of ambitious, and successful global accelerator projects and scientific discoveries that have rewritten the particle physics textbooks.

At CERN for more than fifty years, scientists from all around the world have peacefully been working together to develop new technologies and the necessary infrastructure for answering some of the most puzzling questions of mankind, greatly advancing technologies and scientific understanding in parallel. Can we afford not to prepare a future for the next generation?

\section{The discovery of the Higgs boson was only the beginning}

It is often stated that the Higgs boson concludes a long-standing effort of describing the building blocks that make up our Universe. However one should not neglect the peculiarity of the discovered boson per se. The Higgs is a unique particle - starting already from its rather low mass - and its properties could be a portal to new physics. It is a keystone of the Standard Model and everything we learn about it may be linked to the deepest laws of nature.

There are two complementary paths that should be further explored: precision measurements of the Higgs properties where new physics can manifest itself as tiny deviations from Standard-Model predictions, and searches for new particles and new processes beyond the Standard-Model.

A $100 \mathrm{TeV}$ proton-proton collider could produce conclusive answers to various open questions surrounding the Higgs boson and, in addition, solve some of the most tantalizing puzzles related to the matter-antimatter asymmetry, to dark matter and to dark energy [2]. This machine would offer a bold leap into completely uncharted territory, probing energy scales where fundamentally new physics principles might come into play.

In addition, a future circular electron-positron collider reaching c.m. energies up to $350 \mathrm{GeV}$ (serving as W, Z, Higgs and top factory), will allow for the highest precision measurements ever, of many of the key parameters of the Standard Model [3]. These high-precision measurements in the clean environment of a lepton machine could unveil rare processes and deliver hints for new physics.

Finally, the potential of heavy-ion physics [4] at the hadron collider, and the additional option of hadron-lepton collisions illustrate the enormous versatility and richness of the FCC facility. This multi-faceted research infrastructure would offer profound insights into how matter and the universe behave under extreme conditions. 
The various scenarios explored within the framework of the FCC study can be compounded into a research continuum well extending through the end of the $21^{\text {st }}$ century. While preparing for this future collider complex, we should heed a lesson from science history: even, or especially, if our presently favoured theories are proven wrong we may discover something unanticipated, and possibly truly revolutionary, as was the case, e.g., with the Geiger-Marsden experiments.

\section{The brave new world}

The development of a future circular $100 \mathrm{TeV}$ collider requires significant advancements of numerous technologies. The FCC study has launched long-term R\&D programmes in partnership with many outstanding research centres and universities. As demonstrated by past endeavours, the effective interplay between fundamental science and technological R\&D has a great transformative potential for our daily lives.

Successful technology R\&D relies on interdisciplinary synergies, taking into account the experience from past and present accelerator projects. Ranging from superconducting technologies to new electronics and from novel coolants to reliability studies, the FCC study develops innovative approaches with a great potential impact on industry and society.

The quest for higher energies requires a new magnet technology. Magnets based on $\mathrm{Nb}_{3} \mathrm{Sn}$ superconductor can reach magnetic fields of $16 \mathrm{~T}$ (almost doubling the magnetic field of the LHC Nb-Ti dipole magnets). Such magnet development is supported by the European Commission through the EuroCirCol project (https://fcc.web.cern.ch/eurocircol). Small $\mathrm{Nb}_{3} \mathrm{Sn}$ test coils at Lawrence Berkeley National Laboratory and at CERN have already achieved dipole fields around 16 Tesla [7]. The HL-LHC itself will include a few tens of real $\mathrm{Nb}_{3} \mathrm{Sn}$ accelerator magnets (though with a field of "only" 11 or 12 Tesla), thus helping advance the technology for a future circular hadron collider.

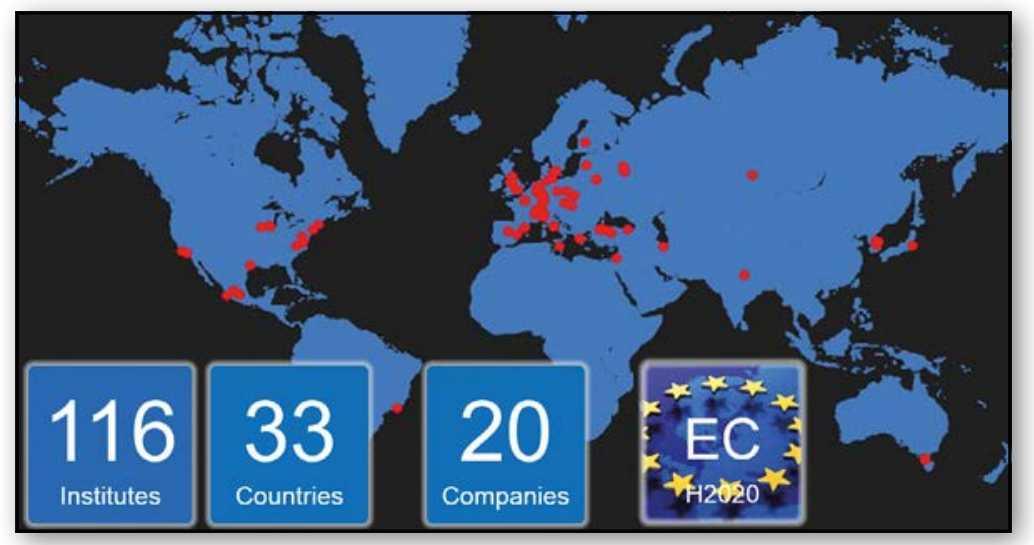

The key element of the future electron-positron collider is a $100 \mathrm{MW}$ RF system [8], for which advanced cavity production techniques and highly efficient RF power sources are being developed. In addition, an ultra-high vacuum and beam screen system [9] for the hadron collider along with a sustainable large-scale cryogenics infrastructure [10] are further examples of key technologies developed in the frame of the FCC study.

The FCC R\&D technology programme is aiming not only at demonstrating the basic feasibility but also at reducing the associated costs and at guaranteeing a sustainable operation. The future circular collider will be much more than a scaled-up version of existing machines.

Fundamental research endeavours often bring about unexpected, life-changing results - as in the case of the basic research that led to the development of magnetic resonance imaging and PET tomography. It was also at CERN that the world-wide-web was invented, changing the lives of billions of people. Present research efforts in superconductivity - to name but one of the many different areas covered by the FCC study - can equally find many life-changing opportunities from transportation to medical applications. Although the benefit of any particular scientific endeavour is unpredictable, there is no doubt that investing in basic research has always paid off over time. Basic science research may transform our world. $\triangle$ FIG. 3:

FCC Collaboration 116 institutes from 33 countries as of June 2017 (Image credit: CERN) brings together

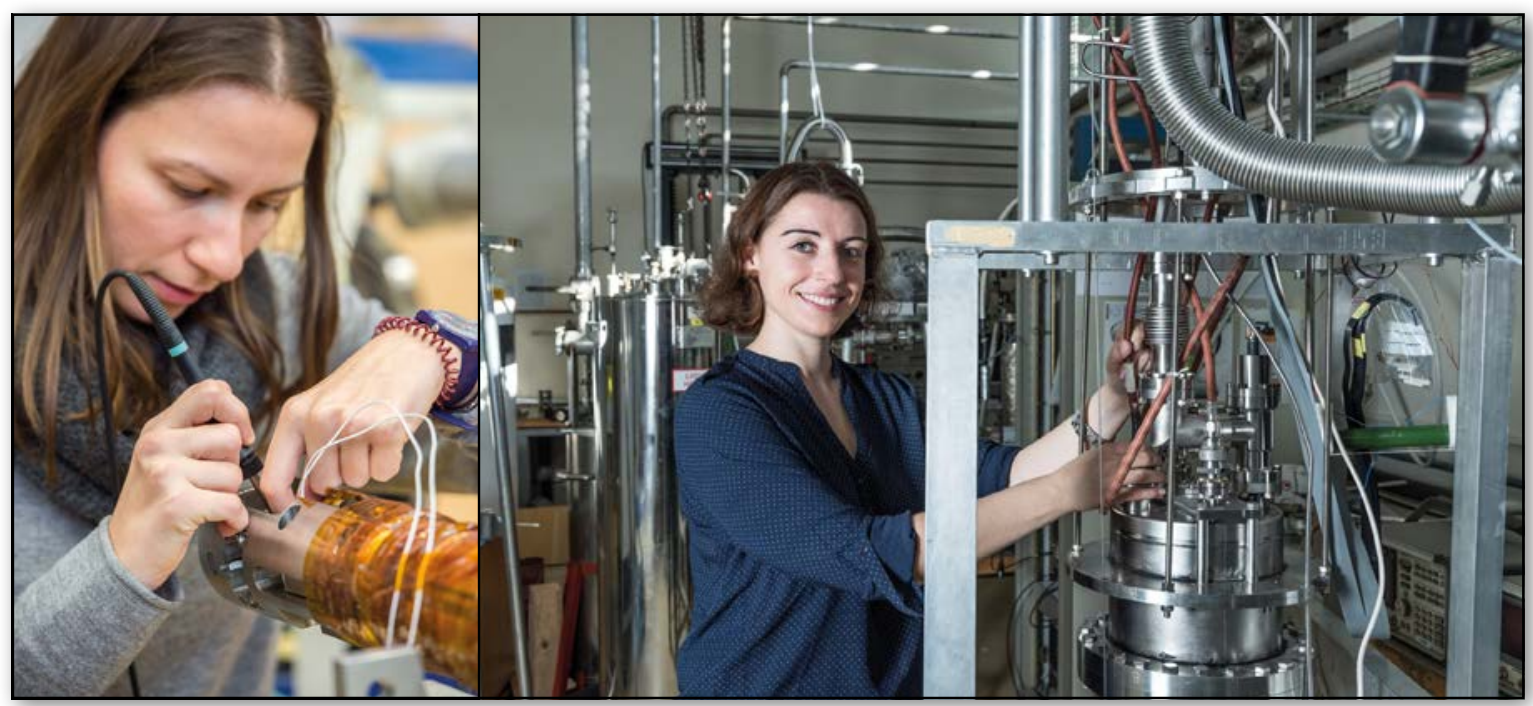

4 FIG. 4: The FCC study offers the opportunity to young researchers from all over Europe to develop now the technologies that will shape our future (Image credit: (left) Robert Hradil \& Monika Majer, (right) CERN) 


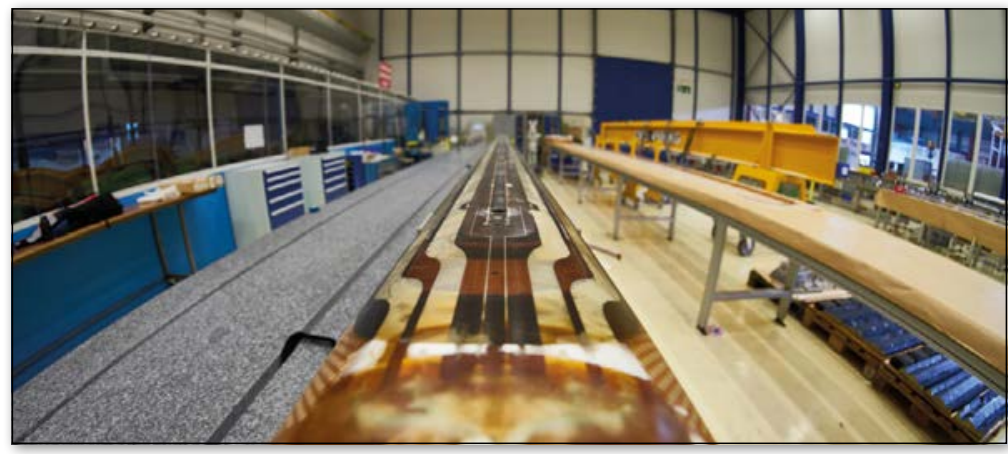

$\triangle$ FIG. 5 .

Photo of the $\mathrm{Nb}_{3} \mathrm{Sn}$ magnet coil that wil

be used in future

high-field magnets

allowing to reach

$12 \mathrm{~T}$ for the High

Luminosity upgrade

of the LHC. The same

material $\left(\mathrm{Nb}_{3} \mathrm{Sn}\right)$

is also studied for

the $16 \mathrm{~T}$ for a future

circular collider

(Image credit: CERN).

v FIG. 6:

First hardware

for FCC: Designing

a novel beam

screen system.

The first prototypes

of a new beam

screen vacuum

system to cope with

the requirements of a $100 \mathrm{TeV}$ collider

are currently

tested. (Image credit: (ERN)

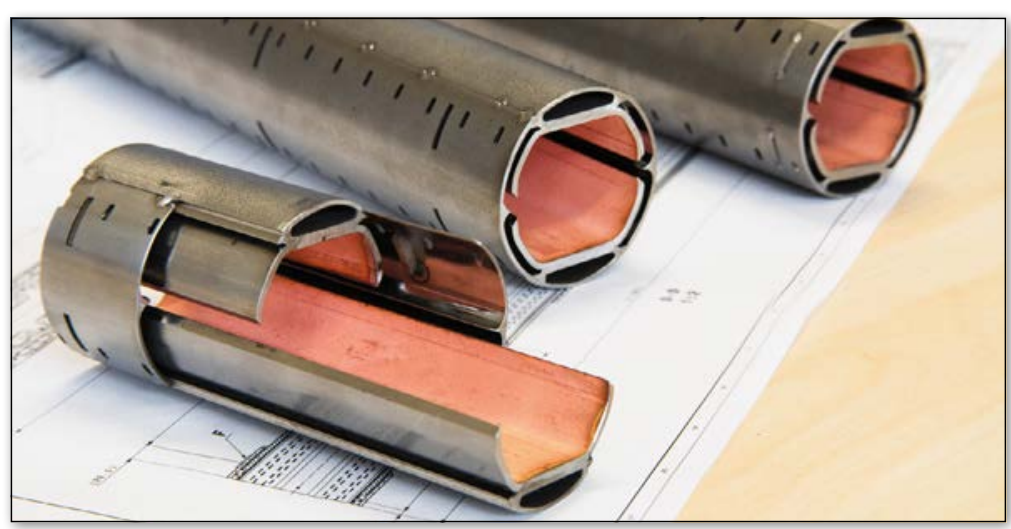
implies investing in fundamental R\&D on novel technologies. The effective collaboration between researchers and industrial partners from an early stage onward is one of the important cornerstones of the FCC study.

Looking at the present physics landscape, we can recognise an ocean of challenges and opportunities not only for physicists, but also for engineers, innovators, and early-stage researchers, and for the future of our societies.
Despite the lack of clear evidence for the next big discovery we can't afford to ignore the many fundamental unanswered questions about the nature of our universe. The future is ours to shape!

\section{About the Authors}

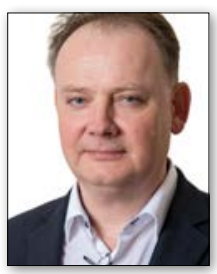

Dr. Michael Benedikt was appointed CERN study leader for the Future Circular Collider (FCC) study in 2013. He started his career with a $\mathrm{PhD}$ on medical accelerator design, as a member of the CERN Proton-Ion Medical Machine Study group. After obtaining his degree, in 1997, he joined CERN's accelerator operation group. He led the PS2 design study from 2005 to 2008 for a new high-performance synchrotron as potential replacement of CERN's Proton Synchrotron. From 2008 until 2013 he was project leader for the design and construction of the accelerator complex for the Austrian hadron therapy centre MedAustron in Wiener Neustadt

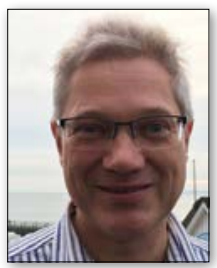

Dr. Frank Zimmermann is the deputy leader of the Future Circular Collider (FCC) study. A senior accelerator scientist at CERN, he also serves as the Editor of the journal "Physical Review Accelerators and Beams" (PRAB). He has earlier worked at SLAC, USA, and DESY, Germany. In 2002 he received the EPS-IGA accelerator prize. He is a fellow of the American Physical Society.

\section{Acknowledgement}

The authors would like to thank all members of the global FCC collaboration for their contributions to the study and Dr. Panos Charitos for his invaluable help in preparing this article.

\section{References}

[1] M. Benedikt, F. Zimmermann, J. Korean Phys. Soc. 69, 893 (2016)

[2] N. Arkani-Hamed, T. Han, M. Mangano, L.-T. Wang, Physics Reports 652, 1 (2016)

[3] M. Bicer, et al., J. High Energ. Phys. 164 (2014). doi:10.1007/ JHEP01 (2014) 164

[4] N. Armesto et al., Nuclear Physics A (2016). https://arxiv.org/ pdf/1601.02963.pdf

[5] M. Benedikt, D. Schulte, and F. Zimmermann., Phys. Rev. ST Accel. Beams 18, 101002 (2015)

[6] K. Oide et al., Phys. Rev. Accel. Beams 19, 111005 (2016)

[7] CERN Courier, Nov 13, 2015, A new record for the RMC test magnet at CERN

[8] E. Cantergiani et al. , Phys. Rev. Accel. Beams 19, 114703 (2016)

[9] R. Kersevan et al., to be published in Proc. ICHEP'16 Chicago

[10] P. Lebrun, L.Tavian, Physics Procedia, $25^{\text {th }}$ International Cryogenic Engineering Conference and the International Materials Conference in 2014, ICEC 25-ICMC 2014 (published December 2015) 